\title{
Una revisión actualizada del concepto de eLearning. Décimo Aniversario
}

\section{An updated review of the concept of eLearning. Tenth anniversary}

\author{
Francisco José García-Peñalvo \\ Departamento de Informática y \\ Automática \\ Universidad de Salamanca \\ fgarcia@usal.es \\ Fecha de recepción: 29-12-2014 \\ Fecha de revisión: 2-3-2015
}

\section{Palabras clave:}

\section{Ecosistemas digitales de aprendizaje; eLearning; mLearning; TIC; LMS; PLE; MOOC; Factor humano; Enseñanza+Aprendizaje; Gestión del conocimiento y de la tecnología.}

\section{Resumen}

Los continuos avances en el plano tecnológico provocan flujos de innovación-aceptaciónconsolidación-obsolescencia propios de las estrategias, ya sean ad hoc o planificadas, de gestión del conocimiento y de la tecnología de las corporaciones, y, a otra escala, de los propios individuos. Los procesos de enseñanza+aprendizaje no son, obviamente, ajenos a esta circunstancia. La irrupción de las Tecnologías de la Información y la Comunicación como herramienta educativa supone un punto de inflexión conceptual y metodológico en la forma en que las instituciones, educativas o no, afrontan los procesos educativos y la gestión del aprendizaje, especialmente en lo tocante al concepto de educación a distancia, que evoluciona, de una manera más o menos significativa, al adoptar Internet como medio,

\author{
Antonio Miguel Seoane Pardo \\ Departamento de Didáctica, Orga- \\ nización y Métodos de Investigación \\ Universidad de Salamanca \\ aseoane@usal.es \\ Fecha de aceptación: 20-3-2015 \\ Fecha de publicación: 27-3-2015
}

Keywords:

Learning digital ecosystems; eLearning; mLearning; ICT; LMS; PLE; MOOC; Human factor; Teaching and Learning; Knowledge and Technology Management.

\section{Abstract}

The continuous advances in technology cause innovation-acceptation-consolidationobsolescence flows regarding the knowledge and technology management strategies, both ad hoc and planned, of the corporations and also, in a different scale, of the individuals. Teaching and learning processes are not obviously unaware of this situation. The irruption of Information and Communication Technologies as educational tools mean both a conceptual and a methodological turning point in the way that institutions, educational or not, face training processes and learning management, especially with regard to the concept of distance education, which evolves, in a more or less significant way, when it adopts Internet as media; that is how the eLearning concept rises. However, from the first eLearning experiences, too much settled 
lo que da lugar al término eLearning. Pero desde las primeras experiencias eLearning muy asentadas en el concepto de plataforma o Learning Management System-, hasta las más recientes, se han producido importantes cambios, de nuevo tanto en el plano tecnológico como metodológico, en los que, además de otros factores, destacan las influencias que los medios sociales producen en los hábitos diarios de los usuarios, de forma que se pone de manifiesto una mayor demanda de personalización del aprendizaje, una conectividad absoluta con otros pares, un acceso ilimitado a los recursos y fuentes de información, una flexibilidad total del modo, el lugar y el momento del acceso y una convivencia cada vez más natural y necesaria de los flujos formales e informales de aprendizaje. Con todo esto, las plataformas eLearning "tradicionales", aunque gozan de un porcentaje altísimo de penetración $\mathrm{y}$ se encuentran sumamente consolidadas, requieren de una evolución y una mayor apertura para dar soporte a este rico abanico de posibilidades que demandan sus usuarios, dejando de ser el centro de atención tecnológica para pasar a ser un componente más en un complejo ecosistema digital orientado a la gestión del aprendizaje y del conocimiento, ya sea institucional o personal. Es, por tanto, necesario volver a hacer una revisión actualizada del concepto de eLearning y releer las definiciones que de este concepto se han aportado desde la experiencia y enfoque de nuestro grupo de investigación GRIAL (GRupo de investigación en InterAcción y eLearning), coincidiendo con el décimo aniversario del artículo "Estado actual de los sistemas e-learning".

\section{Introducción}

Los avances tecnológicos se producen con un ritmo de crecimiento exponencial y con un alcance global que dan lugar a respuestas adaptativas y proactivas en las corporaciones $\mathrm{y}$, a otra escala, en los propios individuos, en función de los flujos de innovación-aceptaciónconsolidación-obsolescencia propios de las estrategias, ya sean ad hoc o planificadas, de on the concept of platform or Learning Management System, up to the present times, there have been significant changes, again in both technological and methodological levels. It is important to underline, among others, the influence of social media in the daily habits of users. This way, an increased demand of learning personalization it is shown, as so as a complete connectivity with other peers, an unlimited access to resources and information sources, a complete flexibility in the way, place and time they access, and a natural and necessary coexistence of both formal and informal learning flows. Thus, the "traditional" eLearning platforms, despite their large penetration and consolidation, need to evolve and open themselves to support this rich fan of possibilities demanded by the users, ceasing to be the centre technological attention to become another component into a complex digital ecosystem oriented to the learning and knowledge management, both at institutional and personal levels. It is therefore necessary to make an updated review of the eLearning concept and its definitions that have been provided from the experience and perspective of our research group GRIAL (Research Group in InterAction and eLearning), coinciding with the tenth anniversary of the "Current status of e-learning systems" paper.

gestión del conocimiento y de la tecnología que se desarrollen. La primera consecuencia directa de esto es que la tecnología modifica los procesos, simplifica y automatiza en diferente grado los flujos de trabajo y provoca cambios en las competencias de las personas. Los procesos de enseñanza+aprendizaje (Illanas \& Llorens, 2011) no son, obviamente, 
ajenos a esta circunstancia. La irrupción de las Tecnologías de la Información y la Comunicación (TIC) como herramienta educativa supone un punto de inflexión conceptual y metodológico en la forma en que las instituciones, educativas o no, afrontan los procesos educativos y la gestión del aprendizaje, especialmente en lo concerniente al concepto de educación a distancia, que evoluciona, de una manera más o menos significativa, al adoptar Internet como medio, lo que da lugar al término eLearning.

Sin embargo, desde estas primeras iniciativas y experiencias de eLearning, las cuales en su mayoría se basaban completamente en las plataformas o Learning Management Systems (LMS), hasta la actualidad, se han producido importantes cambios, tanto en el plano tecnológico como metodológico, en los que, además de otros, destacan las influencias que los medios sociales producen en los hábitos diarios de los usuarios. En este sentido cabe señalar, como demuestran diversos estudios, que la interacción social constituye una de las principales actividades de los usuarios de Internet. Así, de los 3.000 millones de personas que son usuarios de Internet en 2014, con una penetración del 40\% (International Telecommunication Union, 2014), el 74\% de los adultos pertenecen a alguna red social (Pew Research Center, 2014); en España, según datos del Instituto Nacional de Estadística, el $64 \%$ de los internautas participaron en redes sociales en 2013, con un incremento de casi un 5\% con respecto a 2012 (Gimeno, 2014).

Así pues, estos hábitos adquiridos en el uso diario de los medios sociales acaban por trasladarse de una forma $u$ otra a los contextos de aprendizaje, lo que termina por manifestarse en una serie de características como son: a) una mayor demanda de personalización del aprendizaje; b) una conectividad absoluta con otros pares para el desarrollo de actividades formativas; c) un acceso prácticamente ilimitado a los recursos y fuentes de información; d) una flexibilidad total del modo, el lugar y el momento del acceso; y e) una convivencia cada vez más natural y necesaria de los flujos formales e informales de aprendizaje.

Las plataformas eLearning "tradicionales", es decir, los LMS, aunque gozan de un porcentaje altísimo de penetración y se encuentran sumamente consolidadas, pues el $100 \%$ de las Universidades tienen al menos un LMS y el 79,5\% de las grandes corporaciones utilizan estas plataformas en sus iniciativas de formación eLearning (Wexler et al., 2007), empiezan a compartir su protagonismo con una amplia oferta de herramientas, servicios y entornos que, muy ligados a la Web 2.0 (O'Reilly, 2007), permiten configurar ecosistemas educativos que sustentan el concepto de Entorno Virtual Educativo en los que los LMS son un componente opcional más, con lo que se pierde esa concepción por la que los LMS y los Entornos Virtuales Educativos se habían convertido en sinónimos, como ya sucediera en las primeras implantaciones de los LMS (García-Peñalvo \& García Carrasco, 2002), aunque con un enfoque que se orienta hacia los Entornos Personales de Aprendizaje o Personal Learning Environments (PLE) (Adell \& Castañeda, 2010; Attwell, 2007). Estos se ofrecen como la solución a las limitaciones de los LMS en contextos de aprendizaje abiertos y distribuidos centrados en el estudiante (Casquero, 2013), estudiantes que por otra parte lo son en una era digital que les obliga a desarrollar una serie de competencias que les prepare para una vida en continua evolución tecnológica (Hoobs, 2011), entre las que cabría destacar las habilidades para escuchar, buscar informaciones diversas en ecosistemas tecnológicos, interactuar con otros pares constantemente, manejar su identidad digital o producir activamente contenidos, entre otras.

En (García-Peñalvo, 2005) se llevó a cabo una revisión del concepto de eLearning, pero como se ha puesto de manifiesto las plataformas necesitan evolucionar y abrirse para dar soporte a este rico abanico de posibilidades que demandan sus usuarios. Coincidiendo con el décimo aniversario de este artículo, se hace necesario volver a realizar una revisión actualizada del concepto de eLearning y releer las definiciones que de este concepto se han aportado desde la experiencia y enfoque de nuestro grupo de investigación GRIAL (GRupo de investigación en InterAcción y eLearning) (García-Peñalvo, RodríguezConde, et al., 2012). 


\section{Evolución del concepto de eLearning}

Para presentar el estado actual del eLearning es necesario reflejar su evolución en el tiempo. Desde las primeras vinculaciones del concepto de aprendizaje electrónico con las redes de comunicaciones ha existido una tendencia a asimilar eLearning con Educación a Distancia, y, aunque no pueden considerarse sinónimos, esta acepción sigue manteniéndose hoy en día en muchos ámbitos, por lo que se comenzará con una revisión histórica de los hitos más relevantes que han sustentado las primeras formas de educación a distancia. Pero el verdadero foco de este apartado se va poner en la evolución del concepto de eLearning desde su concepción inicial, más ligada a los primeros sistemas software educativos propia de los ordenadores personales, hasta su evolución hacia los sistemas de comunicación que han favorecido el aprendizaje en red y las raíces del conectivismo (Siemens, 2005), especialmente, sin duda, con el punto de inflexión que significa la aparición de la Web, momento a partir del cual la evolución del modelo de eLearning va a estar indisociablemente unido a la evolución de la Web.

Cuando se utiliza una aproximación temporal para presentar la clasificación de los modelos de eLearning en relación con la evolución tecnológica, resulta adecuada la metáfora de la generación (Downes, 2012b; Garrison \& Anderson, 2003; Gros et al., 2009; SeoanePardo \& García-Peñalvo, 2007) o la metáfora de la línea de tiempo (Conole, 2013b) frente a otras taxonomías que hacen uso de otras variables como la centralidad (Anderson, 2008) o el modelo pedagógico subyacente (Anderson \& Dron, 2011), características que, por otra parte, acaban apareciendo ligadas a la evolución tecnológica.

Uno de los primeros trabajos que toman en cuenta la metáfora de lageneración es (Garrison \& Anderson, 2003), donde se hace referencia a cinco etapas o generaciones de eLearning, cada una de ellas con sus diferentes modelos teóricos de referencia. La primera generación se basa en una aproximación conductista. La segunda generación surge por la influencia de nuevas tecnologías de masas y una aceptación creciente de la teoría cognitiva, con estrategias que ponen el énfasis en el concepto de estudio independiente por parte del usuario. La tercera generación, sobre la base de las teorías constructivistas, se centra en las ventajas que ofrece la interacción humana, tanto de forma síncrona como asíncrona. La cuarta y la quinta de las generaciones mencionadas en esta obra de 2003 carecían de un marco teórico de referencia y, de hecho, sus autores consideraban que la mayoría de los programas de formación no cumplían las características de la cuarta generación, que combinaría los tres grandes atributos de la red: extracción de grandes volúmenes de contenidos, capacidad interactiva de la comunicación mediada por ordenador y poder de procesamiento distribuido localmente a través de programación asistida por ordenador. Tampoco existe dicho marco para la quinta generación, basada en un modelo de aprendizaje inteligente y flexible.

En (Seoane-Pardo \& García-Peñalvo, 2007) se hacía referencia al concepto de generación en un contexto tecnológico y de desarrollo estratégico e institucional antes que teórico; sin embargo, esta clasificación no hace sino confirmar la existencia de diferencias cualitativas significativas en la evolución de la formación en línea, tras las cuales es posible entrever el rastro de diversas concepciones de carácter epistemológico o teórico (SeoanePardo, 2014). Así, la primera generación se caracteriza por el desarrollo e implementación de infraestructuras tecnológicas y la búsqueda de herramientas de comunicación más eficientes. Es el producto de un enorme esfuerzo de digitalización de contenidos formativos que, en muchos casos, no constituye sino una mera transposición de los materiales utilizados en contextos presenciales para su despliegue en formatos en línea. Durante esta primera fase no existe un especial interés por los modelos de interacción; es, por tanto, una época de mera fascinación tecnológica. 
La segunda generación se caracteriza por el desarrollo de modelos e indicadores de calidad, a los que han de adaptarse tanto las aplicaciones como los contenidos (Morales, García-Peñalvo, \& Barrón, 2007; Morales, Gómez-Aguilar, \& García-Peñalvo, 2008), y también los componentes humanos de la formación, ya sean docentes o estudiantes. A la vista de que las estrategias y fundamentos epistémicos de base ya habían demostrado su escasa efectividad durante la generación anterior, se trata de reorientarlas mediante la definición de nuevos objetivos y concepciones del aprendizaje. Cada institución, pues, trata de construir algo así como su identidad online, con el propósito de dotarse a sí misma de un cierto modelo o criterio de calidad específico para la formación virtual. Una segunda generación avanzada se basa en la consciencia del valor que posee el factor humano como elemento de calidad (Seoane-Pardo, 2014). La interacción se convierte en condición sine qua non y las iniciativas de formación basadas en el auto-aprendizaje pierden valor frente a las que prometen interacción $y$, especialmente, soporte de tutores (García-Peñalvo \& Seoane-Pardo, 2014; Seoane-Pardo \& GarcíaPeñalvo, 2008). En efecto, surge la necesidad de capacitar adecuadamente a estos nuevos docentes, cuyas atribuciones no coinciden con las propias de la formación presencial, ni con los modelos (virtualmente inexistentes) de docente online de las generaciones anteriores. Por último, se adelanta una hipotética y futura tercera generación, basada en soluciones tecnológicas inteligentes y adaptativas (Berlanga \& García-Peñalvo, 2005, 2008), para la búsqueda y el análisis semántico (Berlanga \& García-Peñalvo, 2004; García-Peñalvo, Berlanga, Moreno, García-Carrasco, \& Carabias, 2004), tanto de recursos e información como de la interacción que se produce en sus iniciativas formativas, que tratan de facilitar el proceso de toma de decisiones adecuadas para una gestión óptima del aprendizaje de todos los estudiantes, tanto individual como colectivamente, mediante la utilización de las estrategias más adecuadas a cada circunstancia, dotando a la experiencia formativa de una extraordinaria flexibilidad metodológica y didáctica y contribuyendo a que el aprendizaje adquiera significatividad y sentido pleno en los intereses y la propia vida del receptor.

(Gros et al., 2009) presentan también tres generaciones, cada una de las cuales se corresponde con un modelo de eLearning diferente. La primera generación se asocia a un modelo centrado en los materiales, donde se incluye el uso de contenidos en papel enriquecidos con formatos digitales que, por lo general, reproducen la estructura clásica propia de los libros. Como herramientas de soporte a la docencia se implementan soluciones de audio y videoconferencia, junto a otros productos software para el apoyo a la formación. La segunda generación se basa en un modelo centrado en el aula virtual, de forma que los entornos virtuales de aprendizaje inspirados en la metáfora del aula son el principal componente y se potencia el uso del streaming de vídeo, con la intención de replicar las dinámicas del aula física. En esta segunda etapa se producen grandes cantidades de materiales en línea, que se suman a la disponibilidad creciente de un buen número de recursos en Internet, los conocidos como objetos de aprendizaje (Morales, Gil, \& García-Peñalvo, 2007; Wiley, 2002). Comienzan las dinámicas de interacción con el uso de sistemas de mensajería $\mathrm{y}$, fundamentalmente, con los foros de discusión. La tercera generación se caracteriza por un modelo centrado en la flexibilidad y la participación, donde los contenidos en línea son más especializados y se combinan contenidos creados tanto por la institución como por los estudiantes. Además se incorporan herramientas orientadas a la reflexión, como los e-porfolios o los blogs (Tan \& Loughlin, 2014), y se busca enriquecer la experiencia de aprendizaje mediante actividades más interactivas como pueden ser juegos, simulaciones, etc., todo ello muy orientado al modelo de comunidades de aprendizaje (Wenger \& Snyder, 2000) y al despliegue en soportes multidispositivo, lo que enlaza perfectamente con el desarrollo de soluciones mLearning (Sánchez Prieto, Olmos Migueláñez, \& García-Peñalvo, 2014).

Para Stephen Downes (2012b) hay un conjunto de tecnologías que han caracterizado el desarrollo del eLearning con el paso de los años que han dado forma al eLearning 
que existe hoy en día y que pueden ayudar a entender la dirección que tomará en un futuro. En primer lugar habla de una generación cero que representa la idea de publicar contenido en línea, incluyendo cualquier elemento multimedia y con una idea muy clara de aprendizaje programado basado en las secuencias de contenidos y actividades determinadas por las elecciones del estudiante y los resultados de ciertas interacciones, como por ejemplo superar un test. Para Downes no se ha avanzado mucho en esta idea fundacional $\mathrm{y}$, de hecho, este ha sido el punto de partida para los subsiguientes desarrollos en el campo del eLearning. La primera generación consiste en la idea de red en sí, herramientas como el correo electrónico, las listas o el gopher permitían una conexión y una comunicación virtual mediante dispositivos hardware y software especializados. Con el desarrollo de la primera generación se produce una maduración de la generación cero y el ordenador personal se convierte en una herramienta educativa para acceder a contenido, pero también para crear y almacenar contenido propio. La segunda generación, que tiene lugar a principios de la década de 1990, viene de la mano del uso de los juegos para uso educativo, permitiendo que muchas personas pudieran ocupar el mismo espacio virtual para comunicarse e interactuar. La tercera generación viene de la mano de las plataformas de aprendizaje o LMS, como aplicaciones que llevaban y aplicaban las ideas y funcionalidades de la generación cero (creación y gestión de contenidos) a la plataforma estrella de la primera generación, la Web. Muchos de los debates sobre el eLearning de esta generación estaban centrados en si el aprendizaje en línea debería basarse más en contenidos (propio de la generación cero) o en la interacción (propio de la primera generación). Los LMS favorecieron la educación a distancia, y de hecho con una aproximación centrada en los contenidos y relegando la interacción a un segundo plano, el eLearning no era más que un tipo especial de educación a distancia que usaba la Web como plataforma de despliegue de contenidos. Sin embargo, se debe reconocer el valor y las aportaciones de los LMS al desarrollo del eLearning. En primer lugar contribuyeron a que los contenidos fueran más modulares, alejándose de la metáfora del libro de texto y facilitando la ubicuidad de los mismos. Además, se fundamentó la idea de que los contenidos pudieran compartirse y reutilizarse, gracias a la metáfora de los objetos de aprendizaje. Pero lo más importante fue reunir en el mismo espacio virtual contenidos y herramientas de comunicación e interacción. La cuarta generación viene promovida por avances ajenos al mercado de los LMS, relacionados con lo que se ha denominado genéricamente como Web 2.0 (O'Reilly, 2007), y que en el campo del eLearning se denominó eLearning 2.0 (Downes, 2005). Los mayores fenómenos de la Web 2.0 fueron el auge de las redes sociales y la creación de contenidos y servicios que podían interactuar con dichos medios sociales. De hecho, aunque la Web 2.0 se describe como una plataforma tecnológica, sería más propio entenderla como la interacción de red aplicada los datos (o los datos aplicados a la interacción de red). El eLearning 2.0, según Downes, aporta importantes aspectos, entre los que cabe citar la creación de un grafo social que define comunicaciones masivas en las que las personas, y no los ordenadores, son los nodos de la red que forma este grafo social; la capacidad de publicación personal que facilita a los participantes de esta red de aprendizaje crear y aportar contenidos a la propia red; la idea de interoperabilidad, tanto en forma de sindicación de contenidos, pero especialmente a través de interfaces de servicios (Alier Forment, Casany Guerrero, Conde González, García-Peñalvo, \& Severance, 2010; Casany et al., 2012; García-Peñalvo, Conde, Alier, \& Casany, 2011); y el concepto de plataforma independiente y ubicua, lo que convierte al eLearning 2.0 en un catalizador fundamental para el mLearning (Conde, García-Peñalvo, Alier, Casany, \& Piguillem, 2013), ya que la Web 2.0 está orientada tanto a los dispositivos móviles como a la interacción social, que se ve muy desarrollada por el acceso desde los teléfonos móviles a las redes sociales. La quinta generación viene caracterizada por la comercialización de servicios web 2.0, la consolidación del marcado de los gestores de contenidos y plataformas de eLearning, la mejora considerable de los sistemas de 
videoconferencia, la computación en la nube (cloud computing) (García-Peñalvo, Alier, \& Lytras, 2012) y los contenidos en abierto, con su influencia en el movimiento y el conocimiento en abierto en general (Fidalgo Blanco, Sein-Echaluce Lacleta, Borrás Gené, \& García-Peñalvo, 2014; García-Peñalvo, García de Figuerola, \& Merlo, 2010a, 2010b) y más particularmente en la educación en abierto (Giaconia \& Hedges, 1982). La sexta generación está completamente centrada en el modelo de los Cursos Online Masivos en Abierto (COMA, del inglés MOOC - Massive Open Online Course) (García-Peñalvo, Fidalgo Blanco, \& Sein-Echaluce Lacleta, 2014; Kay, Reimann, Diebold, \& Kummerfeld, 2013; SCOPEO, 2013; Sonwalkar, 2013b). Los MOOC permiten llevar los efectos de "en abierto" y "de forma gratuita" a una audiencia muy amplia, que puede llegar a ser masiva. Sin embargo, esto no es en sí tan novedoso como para significar una nueva generación. Lo que realmente aportan los MOOC para convertirse en el catalizador de un cambio en la formación online viene de la conjunción de, en primer lugar, la idea de la tecnología distribuida porque las actividades no tienen lugar en un lugar centralizado sino que se distribuyen a través de una amplia red de sitios individuales y servicios, y, por otra parte, la idea del conocimiento distribuido y del aprendizaje que se logra de acuerdo a este concepto, que cambia también la forma en cómo se enseña en red, es decir, no se puede afrontar como una mera secuencia de contenidos (visión conectivista de los MOOC que da lugar a los denominados cMOOC (Fidalgo Blanco, Sein-Echaluce Lacleta, \& García-Peñalvo, 2013)). De acuerdo a esta segunda idea, los MOOC representan la instanciación de los cuatro principios principales de los sistemas distribuidos efectivos: autonomía, diversidad, apertura e interactividad.

Gráinne Conole (2013b, 2014) utiliza la metáfora de la línea de tiempo para introducir los desarrollos tecnológicos claves que han aparecido en los últimos 30 años y que han tenido una implicación decisiva en la formación en línea. En la Figura 1 se muestra esta línea de tiempo.

Al final de los años 80s aparecen las herramientas de autor para crear recursos multimedia, las cuales tuvieron una importancia significativa en la educación especialmente con las nuevas formas de discurso educativo que permitía el hipertexto (Ingram, 2000), importancia que se desplazó hacia la Web en cuanto estuvieron disponibles los primeros navegadores, lo que convirtió a la Web en un auténtico elemento disruptivo y transformador para la tecnología educativa (Harle, 2008). El concepto de objeto de aprendizaje surge con la idea de crear un mercado digital educativo, idea que fracasa pero se convierte en el precursor de los recursos educativos en abierto (Littlejohn, 2003).

Los LMS surgen en 1995 y son significativos porque hacen que las instituciones educativas comiencen a tomar conciencia de que las tecnologías ya no pueden considerarse como innovaciones periféricas, sino que deben ser parte de los servicios nucleares que se le deben de ofrecer a los estudiantes, además los LMS ofrecen a los profesores un contexto seguro con el que pueden experimentar la publicación en línea de contenidos digitales y el uso de herramientas de interacción con sus estudiantes (Conole, 2014). La primera generación de dispositivos móviles surge en la segunda mitad de los 90 con la promesa de facilitar el aprendizaje en cualquier lugar y en cualquier momento (Sharples, Corlett, \& Westmancott, 2002), pero no es hasta que, con la segunda generación de dispositivos móviles (smartphones, tablets y ebooks), se ha dado un paso significativo hacia la madurez y la penetración del mLearning (Conde, Muñoz, \& García-Peñalvo, 2008; Sánchez Prieto et al., 2014). El diseño de aprendizaje surge como una medida contrapuesta al diseño instruccional (Reigeluth \& Carr-Chellman, 2009). El diseño de aprendizaje se orienta más a apoyar e informar de un proceso de diseño pedagógicamente eficaz y que hace un uso adecuado de las tecnologías. Hay tres características importantes del diseño de aprendizaje: la orientación, la visualización y el intercambio (Conole, 2013a). Sobre el año 2000 la gamificación y el aprendizaje basado en juegos toma importancia. Los videojuegos han cambiado la forma en la que los jóvenes conciben la realidad y se relacionan entre 


\begin{tabular}{l|l}
$80 s$ & Recursos multimedia \\
93 & La Web \\
94 & Objetos de aprendizaje \\
95 & LMS \\
98 & Dispositivos móviles \\
99 & Diseño del aprendizaje \\
00 & Gamificación y aprendizaje basado en juegos \\
01 & Recursos educativos en abierto \\
04 & Medios sociales \\
05 & Mundos virtuales \\
07 & eBooks, tablets y smartphones \\
08 & Mooc \\
10 & Analíticas de aprendizaje \\
& Figura 1. La línea de tiempo del eLearning \\
& (adaptado de (Conole, 2014))
\end{tabular}

ellos (McGonigal, 2011; Turkle, 2011). La industria de los videojuegos es una de las de mayor facturación y que mayor crecimiento ha experimentado en los últimos años. Además los videojuegos se utilizan en la enseñanza en lo que se denomina aprendizaje basado en juegos (game-based learning) y como objetos de aprendizaje (videojuegos educativos o serious games) (Prensky, 2006, 2007). Pero una de las últimas tendencias emergentes es la aplicación de las técnicas y las herramientas utilizadas en el diseño de los videojuegos a ámbitos distintos al ocio, en lo que se conoce como gamificación (Werbach \& Hunter, 2012), siendo su aplicación a la docencia una de las líneas más prometedoras (Kapp, 2012). Los jugadores voluntariamente invierten mucho tiempo en el desarrollo de sus habilidades en resolución de problemas en el contexto de los juegos, de forma que se puede utilizar esta capacidad de atracción de los juegos y estas capacidades de los jugadores para sacarles provecho en el proceso de enseñanza+aprendizaje (Illanas et al., 2014). Los recursos educativos en abierto u Open Educational Resources (OER) (UNESCO, 2012) se definen como materiales digitales que se ofrecen libremente y de forma abierta para profesores, estudiantes y autoaprendizaje para su uso y reutilización en docencia, aprendizaje e investigación (OECD, 2007). Tienen su primera iniciativa organizada y difundida a nivel mundial con el OpenCourseWare (OCW) (Open Education Consortium, 2015), que arranca cuando el Instituto Tecnológico de Massachusetts (MIT) anuncia en 2001 que dará acceso gratuito a sus materiales educativos en forma de asignaturas, correspondientes a sus estudios universitarios. No obstante, su eclosión se produce en 2002, promocionado por organizaciones como la UNESCO o la William and Flora Hewlett Foundation, dando lugar al movimiento OER, que hereda los principios y las libertades del software libre y que, de forma progresiva, va definiendo una serie de necesidades, como son la integración entre herramientas (sistemas eLearning, software social, sistemas de gestión de contenidos y herramientas de desarrollo), contenidos y recursos (licencias, buenas prácticas, etc.) desde el punto de vista técnico (Margulies, 2004); tener en cuenta las características técnicas, las características sociales y el área de conocimiento del propio recurso (Centro de Nuevas Iniciativas, 2008); y la reutilización de los recursos en una gran variedad de situaciones de aprendizaje (Wiley, 2008). Los medios sociales cambiaron la naturaleza unidireccional y estática de la Web para convertirla en un medio de dos dimensiones e interactivo, en el que la participación de los usuarios es la clave. Conole y Alevizou (2010) clasifican los medios 
sociales 2.0 utilizados con fines educativos según su funcionalidad en: medios para compartir, medios para realizar mash ups, herramientas de mensajería instantánea y chats, juegos en línea y mundos virtuales, redes sociales, blogs, marcadores sociales, sistemas de recomendación, wikis y herramientas de edición colaborativa, y herramientas de sindicación de contenidos. Estos medios sociales dan lugar a lo que Downes (2005) denomina eLearning 2.0 y que provoca la evolución de muchos LMS para soportar el concepto de aprendizaje social (Conde et al., 2014). Los mundos virtuales, como espacios interactivos en los que varias personas pueden acceder e interaccionar simultáneamente representados por un avatar 3D (García-Peñalvo, Cruz-Benito, \& Therón, 2014), suscitaron mucho interés por su potencial para facilitar aprendizaje basado en roles, gracias a los beneficios que aportan en aspectos como simulación, representación física de construcciones lógicas, entornos mediados, uso de roles y creatividad. Sin embargo, los mundos virtuales no han tenido un uso demasiado exhaustivo, quizás en parte debido a que tienen su dificultad de uso y se han intentado utilizar para objetivos educativos que pueden atenderse de manera más sencilla y efectiva mediante otras tecnologías, por más que hay casos en los que su uso educativo ha sido interesante y exitoso (Allison \& Miller, 2012; Baker, Wentz, \& Woods, 2009; Pizarro Lucas, Cruz-Benito, \& Gil Gonzalo, 2013; Warburton, 2009), destacando, por ejemplo, en el área de la salud (Beard, Wilson, Morra, \& Keelan, 2009; García-Peñalvo, Cruz-Benito, Maderuelo, Pérez-Blanco, \& Martín-Suárez, 2014; Melús-Palazón et al., 2012; Patel, Aggarwal, Cohen, Taylor, \& Darzi, 2013). El término MOOC se define por primera vez en 2008 para describir el curso Connectivism and Connected Knowledge desarrollado por George Siemens y otros (http://cckno8. wordpress.com), lo que dio origen a los denominados posteriormente cMOOC, donde la "c" indica que el curso está basado en pedagogía conectivista (Downes, 2012a; Siemens, 2005). Un segundo tipo de MOOC, que surge en 2011, recibe el nombre de xMOOC, y está basado en contenidos digitales y que hace un mayor énfasis en un aprendizaje individualizado, en contraposición a la postura de los cMOOC más basados en un aprendizaje colaborativo. Actualmente existe un gran interés por el fenómeno MOOC en la comunidad del eLearning (Chiappe Laverde, Hine, \& Martínez Silva, 2015; López Meneses, Vázquez-Cano, \& Román Graván, 2015; Martínez Abad, Rodríguez Conde, \& García-Peñalvo, 2014), pero también es cierto que hay una importante controversia sobre los MOOC (Nkuyubwatsi, 2013; Zapata-Ros, 2013). Por un lado se destaca su papel en la sociedad del conocimiento, ya que proporcionan a cada estudiante la oportunidad de participar y personalizar su entorno de aprendizaje, pero suelen carecer de una realimentación constructiva y de un pensamiento creativo y original (Bates, 2012; Guàrdia, Maina, \& Sangrà, 2013); por otro lado, presentan unas muy bajas tasas de terminación con éxito de los mismos (Belanger \& Thornton, 2013; Daniel, 2012; Jordan, 2013), y se atribuye este fracaso de la supuesta metodología de los MOOC, a la temática, a la heterogeneidad, a la curiosidad que despiertan en personas que no tienen intención real de realizar el curso, etc. (Aguaded Gómez, 2013). Para intentar paliar estos problemas hay trabajos (García-Peñalvo, Fidalgo Blanco, et al., 2014) que se posicionan por combinar las propuestas cMOOC y xMOOC (Castaño Garrido, Maiz, \& Garay Ruiz, 2015; Fidalgo Blanco, Sein-Echaluce Lacleta, et al., 2013; Fidalgo Blanco, SeinEchaluce Lacleta, \& García-Peñalvo, 2015), por introducir características adaptativas (Fidalgo Blanco, García-Peñalvo, \& SeinEchaluce Lacleta, 2013; Rapp, 2012; Sonwalkar, 2013a), o por apostar por la creación de comunidades de aprendizaje (Alario-Hoyos et al., 2013). Esta dicotomía entre cMOOC y xMOOC se pone en duda por varios autores, porque es en sí misma muy limitante y no permite tener en cuenta muchas de las características que muchos MOOC están incorporando actualmente. Así, Lisa Lane (2012) propone un tercer tipo, los sMOOC (skill MOOC), basados en tareas; Stephen Downes (2013) sugiere cuatro criterios para describir la naturaleza de los MOOC (autonomía, diversidad, apertura e 
interactividad); Donald Clark (2013) señala una taxonomía de ocho tipos de MOOC, indicando que pueden situarse en cualquier punto del espectro de los tradicionales cursos online (transferMOOC, madeMOOC, synchMOOC, asynchMOOC, adaptiveMOOC, groupMOOC, connectivistMOOC, miniMOOC); por último, Gráinne Conole (2013c) propone 12 dimensiones para clasificar los MOOC (apertura, masividad, uso de multimedia, densidad de la comunicación, grado de colaboración, itinerario de aprendizaje, aseguramiento de la calidad, grado de reflexión, acreditación, formalidad, autonomía y diversidad). Las analíticas del aprendizaje ha emergido como un nuevo campo de investigación en los últimos años (García-Peñalvo, 2014; Siemens, 2010). Las analíticas del aprendizaje se pueden definir como la medición, recopilación, análisis y presentación de datos sobre los estudiantes y sus contextos, con el propósito de entender y optimizar el aprendizaje y los entornos en que se produce (Ferguson, 2012). Se están desarrollando servicios que recopilan datos de las herramientas educativas para entender y analizar el proceso de aprendizaje de los estudiantes (analítica del aprendizaje) o del propio proceso educativo (analítica académica) (Long \& Siemens, 2011), mostrando los resultados en algún tipo de cuadro de mandos, de forma que la visualización de los datos y la interacción con estas visualizaciones se convierte en una parte de suma importancia en el proceso de análisis y de la toma posterior de decisiones (Gómez, Therón, \& García-Peñalvo, 2009). Gómez Aguilar y otros (2014) han desarrollado un proceso de analítica visual en eLearning (VeLA) que reproduce y extiende el paradigma de Daniel Keim y otros (Keim et al., 2008) aplicado a la analítica educativa, para proporcionar al ciclo analítico una retroalimentación, de forma que queda de la siguiente manera: "Analyze first; Show the important; Zoom, filter and analyze further; Details on demand, Intervention". Este ciclo está representado en la parte central del proceso que se representa en la Figura 2. Se muestra una visión general abstracta de las diferentes etapas (representadas a través de círculos) y sus transiciones (flechas). Dentro de cada etapa se encuentran representadas las dos áreas: analítica de aprendizaje y analítica académica. Como se aprecia en el diagrama, el modelo contempla distintos perfiles de usuario, la diferencia entre estos es el objetivo y, por tanto, el tipo de información de su interés

\section{Proceso de la Analítica Visual en eLearning}

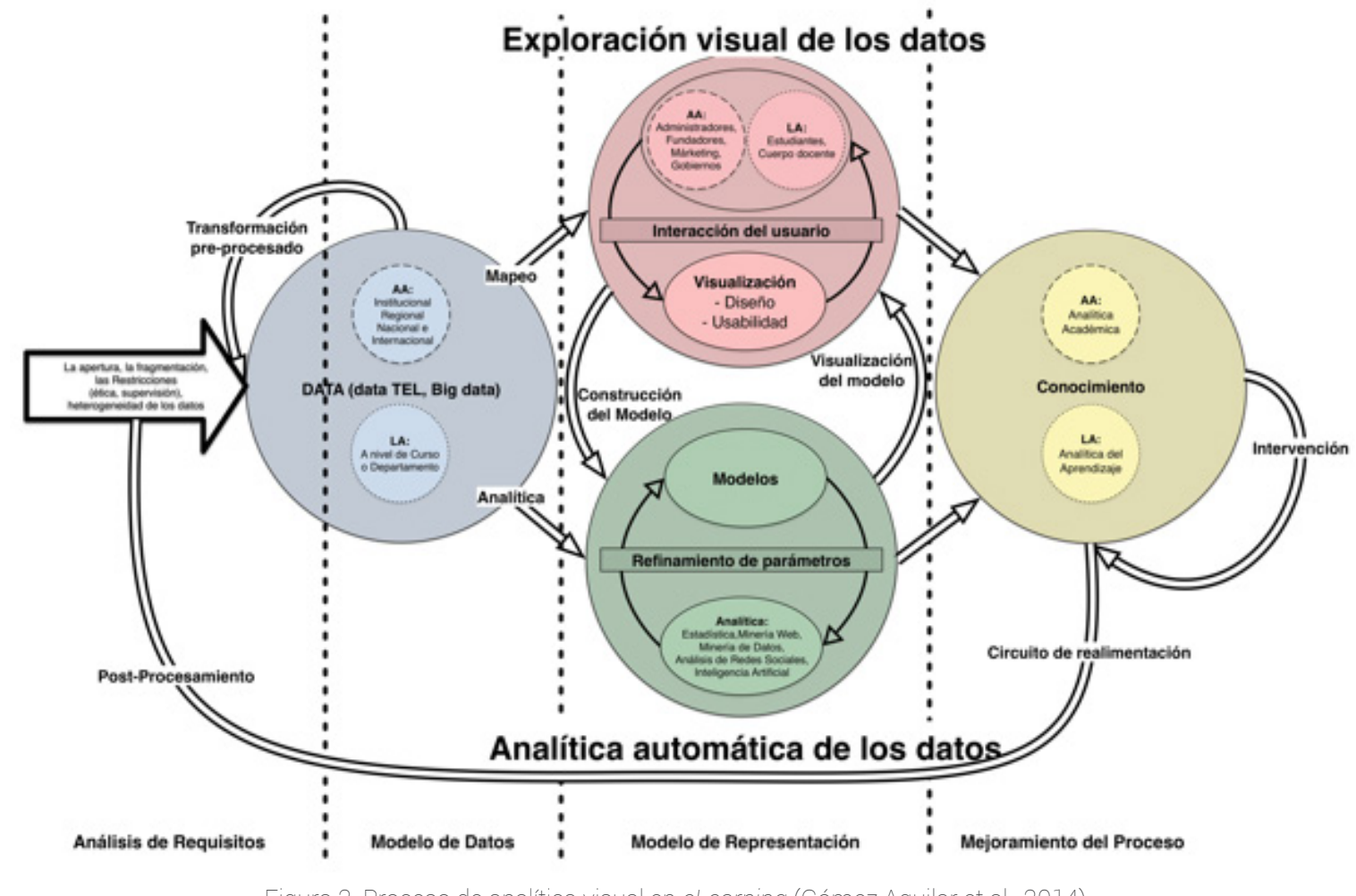




\section{Revisión de la definición de eLearning}

Por la experiencia que se ha desarrollado en el grupo GRIAL, la metáfora de generación es muy adecuada y se comparte en la parte esencial lo que se ha repasado en la sección anterior, especialmente en el sentido expresado por Downes (2012b) de que las generaciones no se sustituyen sino que conviven, y que la madurez de las primeras trae consigo la evolución de las siguientes y la aparición de nuevas generaciones. En este sentido, se cree que el proceso de evolución se puede resumir en tres grandes generaciones que resumirían las diferentes etapas presentadas por los autores referenciados.

La primera generación estaría marcada por la eclosión de plataforma de formación en línea o LMS como evolución de madurez de Entornos Virtuales de Aprendizaje (EVA) (GarcíaPeñalvo \& García Carrasco, 2002) que habían ido surgiendo con la aparición de la Web. Estos LMS están muy centrados en los contenidos digitales y menos en la interacción, y hay más preocupación por el entorno tecnológico que por las connotaciones pedagógicas. Se parte de una experiencia previa en el desarrollo de contenidos multimedia educativos (García-Peñalvo \& García-Carrasco, 2005) y diferentes aplicaciones software educativas (García-Peñalvo, 2002), como fueron el caso de los tutores inteligentes (Corbett, Koedinger, \& Anderson, 1997; Sleeman \& Brown, 1982) y los hipermedias adaptativos (Berlanga, García-Peñalvo, \& Carabias, 2006; Brusilovsky, 2000, 2001).

Alrededor de esta primera generación aparecen algunas de las definiciones más clásicas de eLearning. Así, una de las primeras definiciones se debe a Betty Collis (1996), que definió el "tele-learning" como "la conexión entre personas y recursos a través de las tecnologías de la comunicación con un propósito de aprendizaje". Colin McCormack y David Jones (1998) definen la formación con Internet como "un ambiente creado en la Web en el que los estudiantes y educadores pueden llevar a cabo tareas de aprendizaje. No es solo un mecanismo para distribuir la información a los estudiantes; también supone tareas relacionadas con la comunicación, la evaluación de los alumnos y la gestión de la clase". Trace Urdan y Cornelia Weggen (2000) definen eLearning como "suministro de contenido a través de cualquier medio electrónico, incluyendo Internet, intranets, extranets, comunicación vía satélite, cintas de vídeo y audio, televisión interactiva y CD-ROM. El eLearning se define de una manera más estricta que la educación a distancia, que también incluiría el aprendizaje basado en textos y cursos realizados a través de correspondencia". Lo llamativo de esta definición es que las autoras consideran al eLearning como diferente del online learning. Mientras que el primero es formación con TIC ("el término eLearning se utiliza como sinónimo de aprendizaje basado en tecnología",p.8), el online learning aparece como sinónimo de Web-Based o Internet-Based Learning y, por ende, como un subconjunto del propio eLearning. Marc J. Rosenberg (2001) pone el acento en el uso de Internet como medio para el desarrollo de soluciones tecnológicas orientadas a la mejora del conocimiento. Así, define eLearning como "el uso de tecnologías Internet para la entrega de un amplio rango de soluciones que mejoran el conocimiento y el rendimiento. Está basado en tres criterios fundamentales: 1. El eLearning trabaja en red, lo que lo hace capaz de ser instantáneamente actualizado, almacenado, recuperado, distribuido y permite compartir instrucción o información. 2. Es entregado al usuario final a través del uso de ordenadores utilizando tecnología estándar de Internet. 3. Se enfoca en la visión más amplia del aprendizaje que va más allá de los paradigmas tradicionales de capacitación". Don Morrison (2003) conceptualiza explícitamente al eLearning como proceso de formación continua en su definición "continua asimilación de conocimiento y habilidades por adultos estimulados por eventos de aprendizaje síncronos y asíncronos (y a veces por resultados 
propios de la gestión del conocimiento) que son creados, distribuidos y captados con el soporte de Internet". Germán Ruipérez (2003) ofrece una de las definiciones de referencia de esta primera generación, incluso con tintes de una evolución hacia una segunda generación, al definir la formación en línea como "enseñanza a distancia caracterizada por una separación física entre profesorado y alumnado -sin excluir encuentros físicos puntuales-, entre los que predomina una comunicación de doble vía asíncrona donde se usa preferentemente Internet como medio de comunicación y de distribución del conocimiento, de tal manera que el alumno es el centro de una formación independiente y flexible, al tener que gestionar su propio aprendizaje, generalmente con ayuda de tutores externos". Al hablar de "separación física" no alude expresamente al concepto de "educación a distancia" convencional. La no concurrencia física en un mismo espacio (y con frecuencia tampoco en un mismo tiempo) no es obstáculo para la combinación de momentos de formación online con otros de carácter presencial, de modo que esta dicotomía presencial-no presencial no se percibe como algo inexorable. En el contexto del grupo GRIAL, y al igual que en la definición anterior cerrando una generación con visos a abrir una nueva generación con más atención a la interacción, se definía

eLearning como "capacitación no presencial que, a través de plataformas tecnológicas, posibilita y flexibiliza el acceso y el tiempo en el proceso de enseñanza-aprendizaje, adecuándolos a las habilidades, necesidades y disponibilidades de cada discente, además de garantizar ambientes de aprendizaje colaborativos mediante el uso de herramientas de comunicación síncrona y asíncrona, potenciando en suma el proceso de gestión basado en competencias" (García-Peñalvo, 2005).

La segunda generación pone el énfasis en el factor humano. La interacción entre pares y la comunicación entre el docente y los discentes supone una seña de identidad de una formación de calidad que se quiere distanciar de la mera publicación de contenidos o de la mera información. Ni que decir tiene que el desarrollo del paradigma de la Web 2.0 va a ser el aliado tecnológico ideal para potenciar esta socialización del aprendizaje y que llevaría a ese eLearning 2.0 (Downes, 2005) que ya se ha mencionado anteriormente. Una mayor interacción produce una mayor cantidad de datos que pueden ser obtenidos y analizados, de nuevo se pone de manifiesto otro de los principios básicos de la Web 2.0 y su relación con los datos y que permitirá su aprovechamiento para la toma de decisiones con los adecuados procesos de analítica de datos. El despliegue de los sistemas móviles y de soluciones $3 \mathrm{D}$ son propias de esta generación en sucesivas iteraciones y según la tecnología va estando más madura. Hay una clara evolución de los LMS para soportar estas características de movilidad, socialización e interoperabilidad a nivel de datos (Conde et al., 2014). Otro elemento que no se puede obviar en esta segunda generación es la influencia del movimiento del Conocimiento en Abierto (García-Peñalvo et al., 2010a, 2010b) en relación tanto con los contenidos como con la tecnología propios de esta generación.

Como ejemplos de definiciones más acordes con esta segunda generación se puede recurrir a la de Ruth Clark y Richard Mayer (2011) en la que definen el eLearning como "formación desplegada un dispositivo digital como un ordenador o un dispositivo móvil con el que se intenta dar soporte al aprendizaje". Esta definición no dista en demasía de la definición de Betty Collis (1996) dictada 15 años antes, si bien esta última no se restringe a la conexión de personas y contenidos, como sí hacía Betty Collis, y deja la puerta abierta a todas las posibilidades que ofrecen los dispositivos digitales, incluidos los dispositivos móviles, siempre que se empleen intencionalmente con propósito formativo, pero reduce una vez más el eLearning al factor tecnológico, por lo que se podría caracterizar como una definición en la transición entre generaciones, que si bien tecnológicamente se sitúa más cerca de la segunda, su olvido del factor humano y de la interacción entre las personas la aleja de los objetivos que, según lo que se defiende desde el grupo GRIAL, conducirían a una formación en línea de calidad. Congruentemente con esto, la definición que desde este grupo de investigación caracterizaría plenamente 
esta segunda generación de eLearning sería "desde una perspectiva de la calidad se puede definir eLearning como un proceso de enseñanza+aprendizaje, orientado a la adquisición de una serie de competencias y destrezas por parte del estudiante, caracterizado por el uso de las tecnologías basadas en web, la secuenciación de contenidos y actividades estructuradas según estrategias preestablecidas a la vez que flexibles, la interacción con la red de estudiantes y tutores y unos mecanismos adecuados de evaluación, tanto del aprendizaje resultante como de la intervención formativa en su conjunto, en un ambiente de trabajo colaborativo de presencia diferida en espacio y tiempo, y enriquecido por un conjunto de servicios de valor añadido que la tecnología puede aportar para lograr la máxima interacción, garantizando así la más alta calidad en el proceso" (García-Peñalvo, 2008).

En primer lugar, la formación en línea es un proceso de enseñanza+aprendizaje (Illanas \& Llorens, 2011). Esto implica, por tanto, que la responsabilidad de la formación es compartida entre los docentes y discentes, en la medida en que surge como fruto de la interacción entre ambos perfiles. Esto, en sí mismo, constituye un elemento diferencial respecto a otras definiciones en las que recae sobre el estudiante la práctica totalidad de la responsabilidad de su formación. Aquí, por el contrario, se proponen la interacción y el trabajo colaborativo como elementos catalizadores del aprendizaje, lo cual, lógicamente solo puede lograrse efectivamente mediante la colaboración de los estudiantes, sí, pero por lo general esta "magia" no adviene sin la presencia de unos perfiles docentes capaces de gestionar los flujos de comunicación y liderar los procesos formativos en su conjunto. Otro de los elementos cruciales consiste en el establecimiento de un difícil equilibrio entre flexibilidad y estructura formativa; debe existir un balance flexible entre la adaptación a las necesidades del estudiante y la exigencia de alcanzar las competencias y destrezas adecuadas. Las estrategias al respecto no pueden ser inflexibles, pero tampoco completamente abiertas y desestructuradas, pues los riesgos de ambos extremos han demostrado ser igualmente fatales para los resultados de la formación. En lo referente a la presencia diferida en el espacio y en el tiempo, esta definición se desmarca explícitamente tanto de la formación a distancia convencional como de otros intentos de replicar en el eLearning contextos propios de la enseñanza presencial $y$, por tanto, supone que la formación online posee características y permite dinámicas propias tanto de la formación presencial como a distancia, si bien todas ellas deben ser replanteadas específicamente para estos contextos en línea, cualitativamente nuevos.

La tercera generación de eLearning se caracteriza por dos aspectos que simbióticamente se influyen.

El primer elemento es tecnológico, se rompe el concepto de plataforma de aprendizaje o LMS como elemento monolítico y único responsable de la funcionalidad para la formación en línea. Desde la aparición de la Web 2.0 y las herramientas de carácter social, el concepto de plataforma se ha quedado limitado y, en su lugar, la tecnología debe servir como un ecosistema tecnológico de aprendizaje (García-Holgado \& GarcíaPeñalvo, 2013, 2014a, 2014b), transcendiendo la mera acumulación de tecnologías de moda (Llorens, 2009, 2011), que permita facilitar al máximo la interacción y ofrecer la mayor flexibilidad didáctica a cualquier docente. Un ecosistema es una comunidad de seres vivos cuyos procesos vitales están interrelacionados y cuyo desarrollo se basa en los factores físicos del medio ambiente. La definición de ecosistema tecnológico varía de unos autores a otros, pero todos están de acuerdo en un punto fundamental, hay una clara relación entre las características de un ecosistema natural y un ecosistema tecnológico en cualquiera de sus variantes (Berthelemy, 2013; Chang \& West, 2006; Chen \& Chang, 2007; Laanpere, 2012; Pata, 2011). Por analogía con esta definición, se propone un ecosistema tecnológico donde una comunidad, con métodos educativos, políticas, reglamentos, aplicaciones y equipos de trabajo pueden coexistir de manera que sus procesos están interrelacionados y su aplicación se basa en los factores físicos del entorno tecnológico (Llorens, Molina, Compañ, \& Satorre, 2014).

El segundo aspecto es la pérdida de la 
verticalidad del concepto de eLearning para convertirse en un elemento más transversal y universal que se pone al servicio de la formación en su sentido más amplio. Tanto desde un punto de vista intencional (formal y no formal) como no intencional (informal) los servicios de los ecosistemas de eLearning se ponen al servicio de las personas involucradas en los procesos de enseñanza+aprendizaje o de autoaprendizaje, integrándose de una forma transparente en los diseños educativos o de las actividades de aprendizaje que se establezcan o se decidan. Se pone de manifiesto la penetración de la tecnología en las vidas cotidianas de las personas, lo que facilita que se eliminen las fronteras, cada vez más difusas, entre el aprendizaje formal e informal (García-Peñalvo, Colomo-Palacios, \& Lytras, 2012).

El concepto de ecosistema tecnológico de aprendizaje sirve para esta universalización del eLearning ya sea para dar un soporte a un contexto institucional (García-Holgado \& García-Peñalvo, 2014a; García-Peñalvo, Johnson, Ribeiro Alves, Minovic, \& CondeGonzález, 2014; Hirsch \& Ng, 2011) o personal a través del concepto, más metafórico que tecnológico, de PLE (Wilson et al., 2007). Estos PLE buscan facilitar el aprendizaje de las personas, al permitir que estas utilicen aquellas herramientas que consideren oportunas para aprender (normalmente con las que están más familiarizados), sin estar vinculados a un entorno institucional concreto o a un período de tiempo específico (Adell \& Castañeda, 2010). Con los PLE el individuo pasa a ser el responsable de su propio aprendizaje, ya que puede gestionar su aprendizaje al determinar qué herramientas a usar, pasando de ser consumidor a proveedor de aprendizaje, además de aprender a relacionarse con otros, pero siempre según sus necesidades específicas, etc. (Schaffert \& Hilzensauer, 2008).

Congruentemente con los elementos que definen esta tercera generación, el Grupo GRIAL propone una nueva definición de eLearning sobre la base de la definición de (Seoane-Pardo, 2014): "proceso formativo, de naturaleza intencional o no intencional, orientado a la adquisición de una serie de competencias y destrezas en un contexto social, que se desarrolla en un ecosistema tecnológico en el que interactúan diferentes perfiles de usuarios que comparten contenidos, actividades y experiencias y que, en situaciones de aprendizaje formal, debe ser tutelado por actores docentes cuya actividad contribuya a garantizar la calidad de todos los factores involucrados".

\section{Un modelo de eLearning expresado como un lenguaje de patrón}

El Grupo GRIAL tiene una experiencia de más de 10 años de investigación y práctica del eLearning, fundamentada en el valor de la interacción humana, a la que tanto la tecnología como los procesos y estrategias deben servir, y que ha condicionado tanto el desarrollo de las soluciones tecnológicas como metodológicas, didácticas y de investigación académica desarrolladas en el grupo, con el firme propósito de lograr una formación que responda a las más elevadas exigencias de calidad y enfocada a la adquisición de competencias y destrezas de la manera más eficiente posible. Esta experiencia se ha formalizado en un lenguaje de patrón (Alexander, Ishikawa, \& Silverstein, 1977) e intenta trasmitir las lecciones aprendidas y las buenas prácticas tras haber impartido más de 22.150 horas, a unos 4.400 destinatarios en más de 150 iniciativas de formación de Grado, Posgrado, Formación Continua y a demanda, en cursos tanto de corta como de larga duración y en contextos de aprendizaje académicos y profesionales. A esta experiencia docente se debe añadir la experiencia en gestión universitaria al haber liderado y gestionado la política del eLearning de una institución como la Universidad de Salamanca durante dos años, hasta crear una comunidad virtual de más 30.000 usuarios entre docentes y profesores, a finales de 2009.

El modelo de eLearning que se ha definido se puede encontrar ampliamente detallado en (Seoane Pardo \& García-Peñalvo, 2014; 
Seoane-Pardo, 2014). Aquí solo se va a describir la arquitectura del lenguaje de patrón, Figuras 3 y 4, para que sirva como guía y resumen de la experiencia acumulada. La arquitectura del modelo incluye todos los elementos esenciales que deben estar presentes en cualquier escenario posible en el que se implementen soluciones tecnológicas y metodológicas de formación en línea. Estos elementos son el plan estratégico $(A 1$. Course Prep.), compuesto por el conjunto de decisiones de carácter institucional o corporativo que afectan a todas las iniciativas formativas que recaen bajo su responsabilidad; la planificación instructiva (A2. Unit Plan), es decir, la programación de la actividad didáctica por parte del docente o los diseñadores instructivos; la acción docente (A3. Tutoring), compuesta por el conjunto de actividades que los docentes llevan a cabo como parte del proceso formativo; los ajustes formativos (A4. Settings), que incluyen el diseño e implementación de las acciones que contribuyen a solventar los problemas de aprendizaje individuales o colectivos que se puedan detectar durante el proceso; por último, la evaluación (A5. Evaluation) es el conjunto de estrategias, instrumentos, métodos y actividades que permiten medir el rendimiento del aprendizaje, valorar la calidad de las acciones formativas y proporcionar mecanismos que favorezcan la mejora de todo el proceso, desde las decisiones institucionales hasta el propio sistema de evaluación en su conjunto. En la imagen de las Figuras 3 y 4 se representan dentro de una elipse y ordenadas por la letra "A" a la que sigue un número, del 1 al 5 .

Un segundo nivel de granularidad está compuesto por los elementos del diseño del sistema. Estos componentes, quese representan en rectángulos que se identifican por la letra "D" y se numeran del 1 al 7, constituyen los diferentes problemas o dificultades en los que se estructura cada uno de los niveles arquitectónicos de los que dependen. Estos dos primeros niveles pretenden representar los problemas, características y acciones a los que se debe enfrentar cualquier institución que desee acometer la tarea de desplegar acciones formativas de eLearning de calidad, tanto desde un punto de vista de las decisiones de carácter estratégico como mediante la acción de los diferentes perfiles profesionales involucrados, especialmente aquellos de carácter docente. Los niveles de arquitectura y diseño se muestran de manera gráfica en la Figura 3.

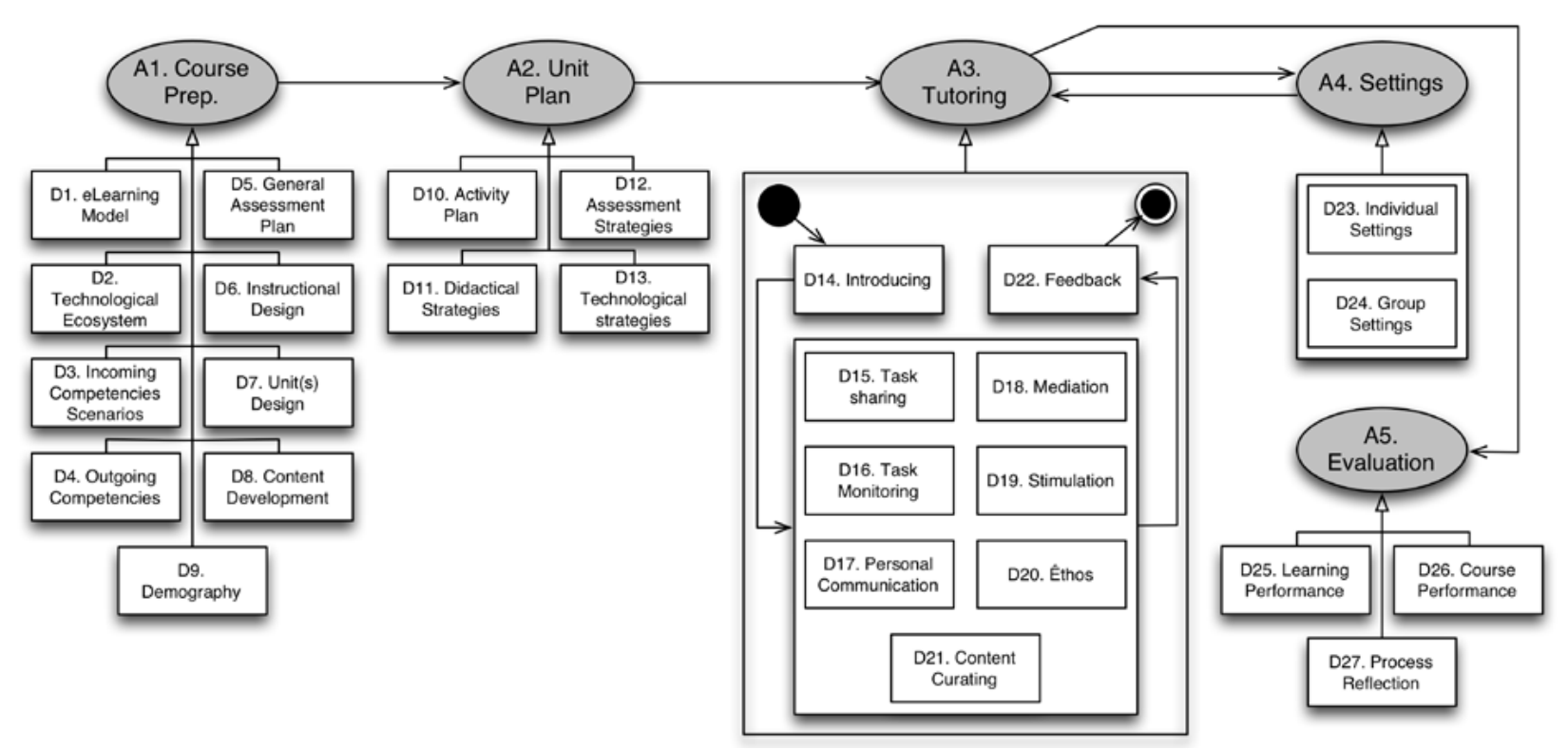


El tercer y último nivel está compuesto por los patrones que proporcionan soluciones concretas o respuestas específicas a los problemas de diseño que se desean resolver dentro de la arquitectura institucional del eLearning de la entidad en cuestión. Estos patrones de bajo nivel o idioms no constituyen soluciones necesariamente exportables, puesto que son fuertemente dependientes del contexto y del modo en que una institución aborda sus problemas de diseño expresados en los patrones del nivel inmediatamente superior. Se han identificado, inicialmente, 58 idioms que constituyen otras tantas respuestas a los problemas de diseño y arquitectura que se han venido observando como fruto de la experiencia recogida en diferentes contextos, y que presuponen una visión del eLearning basada en la importancia del factor humano y de la interacción como elemento esencial del aprendizaje. Estos idioms se representan mediante rectángulos redondeados dependientes de los elementos de diseño, que se denominan mediante la letra "I" seguida de un número, del 1 al 58. Se puede observar una imagen completa de este lenguaje de patrón de GRIAL, compuesto por un total de 90 elementos, en la Figura 4.

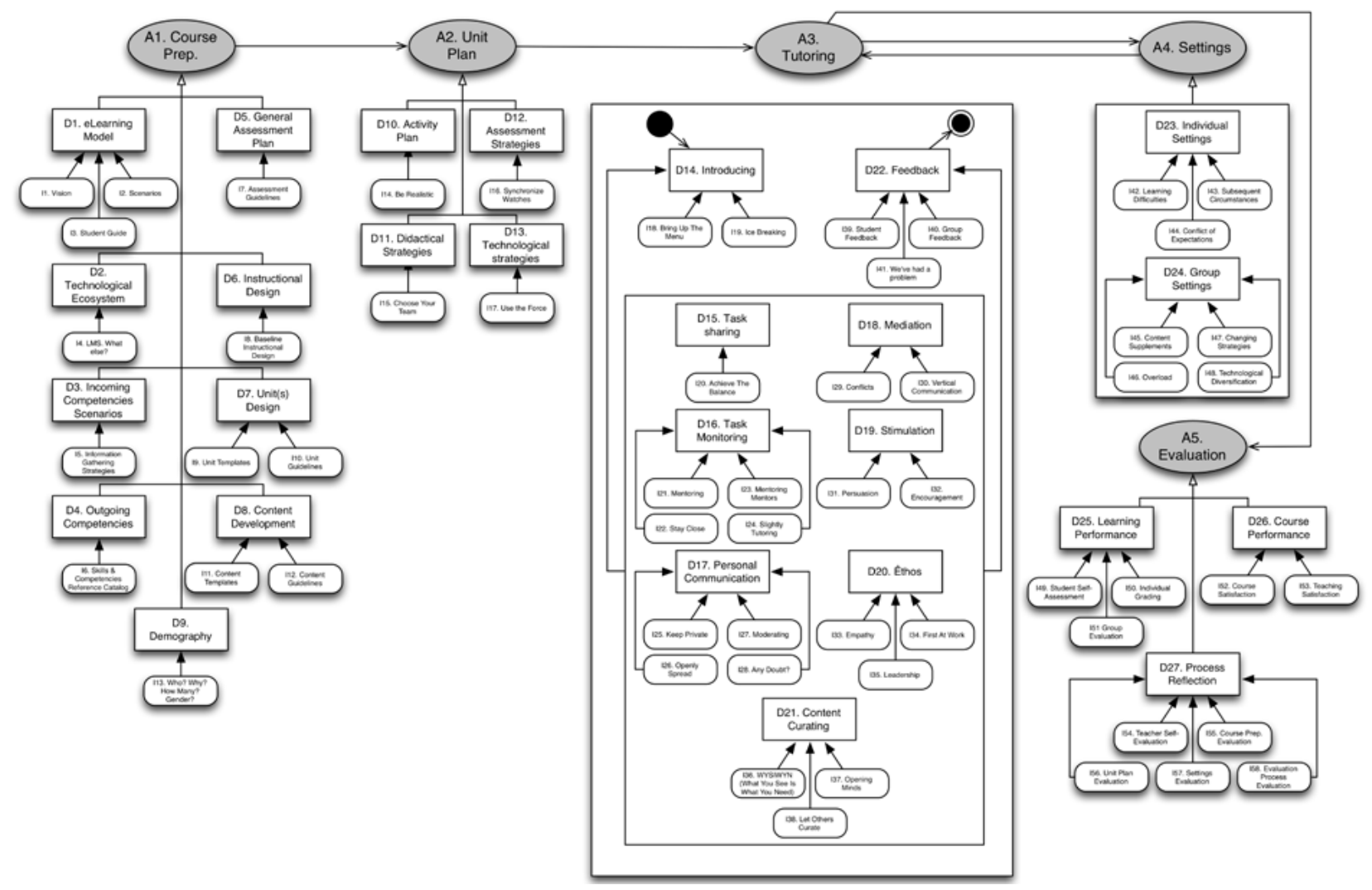

Figura 4. Lenguaje de patrón de eLearning completo (Seoane Pardo \& García-Peñalvo, 2014; Seoane-Pardo, 2014).

\section{Conclusiones}

En este artículo se ha querido hacer un repaso actualizado del concepto de eLearning tras el realizado hace 10 años en (García-Peñalvo, 2005), período en el que han cambiado muchos aspectos, por supuesto tecnológicos, entre los que cabe destacar la pérdida de protagonismo de los gestores de contenidos educativos o LMS, como aplicaciones cerradas y monolíticas sobre las que recaía todo el peso de la gestión tecnológica del proceso formativo, para pasar a ser un componente más de un ecosistema tecnológico mucho más complejo, basado en servicios que interoperan entre sí, y que refleja las necesidades de tanto instituciones como de individuos.

Pero los cambios no solo han venido de la 
mano de la tecnología. El eLearning se ha ido convirtiendo en una herramienta al servicio de los procesos de enseñanza+aprendizaje, de manera que está perdiendo esa concepción binaria por la que una formación era online o no, para integrarse de una forma mucho más transparente en los procesos educativos y de autoaprendizaje en función de las necesidades de los involucrados.

Para hacer este repaso se ha seguido la metáfora de generación de eLearning, haciendo mención a lo que diferentes autores han ido estableciendo a este respecto. En la experiencia desarrollada, se coincide con esta idea de generaciones, pero con el matiz de que las generaciones no se sustituyen unas a las otras, sino que la evolución y la madurez de las anteriores influyen en la creación de las nuevas. En este sentido se presentan tres generaciones, una marcada por la aparición en escena de los LMS muy centrada en estas plataformas y en los contenidos, una segunda marcada por la interacción y el factor humano gracias al desarrollo de la Web 2.0 y a la importancia del conocimiento abierto, $y$, finalmente, una tercera en la que se rompe con la hegemonía de los LMS para ir hacia ecosistemas tecnológicos de aprendizaje

orientados tanto a nivel institucional o corporativo como individual dando soporte a aprendizajes formales e informales.

Puede resultar sorprendente que en las tres generaciones mencionadas no se hayan tomado a los MOOC como elemento disruptivo para protagonizar una generación. Esto es así porque se ha considerado que el modelo MOOC, si bien es importante, tiene diferentes aproximaciones que podrían estar perfectamente recogidas en las características de la primera generación, como sucede con muchos de los xMOOC, o en la segunda y tercera generación para muchos de los cMOOC.

Finalmente, para terminar con este repaso, se ha querido transmitir la experiencia del grupo GRIAL de más 10 años de investigación y aplicación del eLearning que se refleja en el modelo de eLearning GRIAL formalizado en un lenguaje de patrón. Este modelo transmite una concepción del eLearning basada en la importancia del factor humano y de la interacción como elemento esencial del aprendizaje. Sin embargo, al haber formalizado el modelo de eLearning como un lenguaje de patrón, se ha querido facilitar el proceso de "exportación" del modelo a cualquier otro contexto institucional o corporativo. Para esto se debería revisar la definición de los elementos de arquitectura y diseño propuestos para que se puedan ajustar lo más posible al escenario específico. Esta tarea permitiría, por una parte, adquirir conciencia de todos los factores involucrados y sobre los que es necesario tomar decisiones, a la vez que invita a construir una base de conocimiento institucional y a explicitar los principios de carácter estratégico que inspiran la visión o modelo de eLearning que la entidad pretende desarrollar. A continuación es posible revisar y adaptar los dialectos de este lenguaje de patrón al escenario concreto de que se trate, e incluso crear las soluciones de bajo nivel que encajen mejor con la idiosincrasia de la entidad.

\section{Agradecimientos}

Se quiere agradecer el apoyo de todos los miembros del Grupo GRIAL (Grupo de Excelencia de la Junta de Castilla y León -
GR47) que con su trabajo han contribuido a la evolución del grupo y su investigación en estos más de10 años de trabajo intenso. 


\section{Referencias}

Adell, J., \& Castañeda, L. (2010). Los Entornos Personales de Aprendizaje (PLEs): una nueva manera de entender el aprendizaje. In R. Roig Vila \& M. Fiorucci (Eds.), Claves para la investigación en innovación y calidad educativas. La integración de las Tecnologías de la Información y la Comunicación y la Interculturalidad en las aulas. Stumenti di ricerca per l'innovaziones e la qualità in ámbito educativo. La Tecnologie dell'informazione e della Comunicaziones e l'interculturalità nella scuola. Alcoy, Spain: Marfil - Roma TRE Universita degli studi.

Aguaded Gómez, J. (2013). La revolución MOOCs, ¿una nueva educación desde el paradigma tecnológico? Comunicar, 41, 7-8. http://dx.doi.org/10.3916/C41-2013-a1

Alario-Hoyos, C., Pérez-Sanagustín, M., Delgado-Kloos, C., Parada, H. A., MuñozOrganero, M., \& Rodríguez-de-las-Heras, A. (2013). Analysing the Impact of Built-In and External Social Tools in a MOOC on Educational Technologies. In D. HernándezLeo, T. Ley, R. Klamma, \& A. Harrer (Eds.), Scaling up Learning for Sustained Impact. 8th European Conference, on Technology Enhanced Learning, EC-TEL 2013, Paphos, Cyprus, September 17-21, 2013. Proceedings (Vol. 8095, pp. 5-18). Berlin Heidelberg: Springer.

Alexander, C., Ishikawa, S., \& Silverstein, M. (1977). A Pattern Language. Towns, Buildings, Construction. New York, NY, USA: Oxford University Press.

Alier Forment, M., Casany Guerrero, M. J., Conde González, M. Á., García-Peñalvo, F. J., \& Severance, C. (2010). Interoperability for LMS: the missing piece to become the common place for e-learning innovation. International Journal of Knowledge and Learning (IJKL), 6(2/3), 130-141.

http://dx.doi.org/10.1504/IJKL.2010.034749
Allison, C., \& Miller, A. (2012). Open virtual worlds for open learning. St. Andrews, UK: Higher Education Academy.

Anderson, T. (2008). Toward a theory of online learning. In T. Anderson (Ed.), Theory and practice of online learning (2nd ed., pp. 45-74). Edmonton, AB, Canada: AU Press, Athabasca University.

Anderson, T., \& Dron, J. (2011). Three generations of distance education pedagogy. The International Review of Research in Open and Distance Learning, 12(3), 80-97.

Attwell, G. (2007). The Personal Learning Environments - The future of eLearning? eLearning Papers, 2(1).

Baker, S. C., Wentz, R. K., \& Woods, M. M. (2009). Using Virtual Worlds in Education: Second Life® as an Educational Tool. Teaching of Psychology, 36(1), 59-64.

http://dx.doi. org/10.1080/00986280802529079

Bates, T. (2012). What's right and what's wrong about Coursera-style MOOCs. Retrieved from http://www.tonybates.ca/2012/08/05/ whats-right-and-whats-wrong-aboutcoursera-style-moocs/

Beard, L., Wilson, K., Morra, D., \& Keelan, J. (2009). A Survey of Health-Related Activities on Second Life. Journal of Medical Internet Research, 11(2), e17.

http://dx.doi.org/10.2196/jmir.1192

Belanger, Y., \& Thornton, J. (2013). Bioelectricity: A Quantitative Approach. Duke University's First MOOC: Duke University.

Berlanga, A. J., \& García-Peñalvo, F. J. (2004). Towards semantic metadata for learning elements. 
Berlanga, A. J., \& García-Peñalvo, F. J. (2005). IMS LD reusable elements for adaptive learning designs. Journal of Interactive Media in Education, 11.

http://dx.doi.org/10.5334/2005-11

Berlanga, A. J., \& García-Peñalvo, F. J. (2008). Learning Design in Adaptive Educational Hypermedia Systems. Journal of Universal Computer Science, 14(22), 36273647.

Berlanga, A. J., García-Peñalvo, F. J., \& Carabias, J. (2006). Authoring adaptive learning designs using IMS LD. In V. Wade, H. Ashman, \& B. Smyth (Eds.), Adaptive Hypermedia and Adaptive Web-Based Systems, Proceedings (Vol. 4018, pp. 31-40). Berlin: Springer Verlag. http://dx.doi.org/10.1007/11768012_5

Berthelemy, M. (2013). Definition of a learning ecosystem. Retrieved from http://www.learningconversations. co.uk/main/index.php/2010/01/10/ the-characteristics-of-a-learningecosystem?blog $=5$

Brusilovsky, P. (2000). Adaptive Hypermedia: From Intelligent Tutoring Systems to WebBased Education Intelligent Tutoring Systems. 5th International Conference, ITS 2000 Montréal, Canada, June 19-23, 2000 Proceedings (pp. 1-7). Berlin, Heidelberg: Springer.

http://dx.doi.org/10.1007/3-540-44595-1

Brusilovsky, P. (2001). Adaptive Hypermedia, en User Modeling and User-Adapted Interaction. User Modeling and User-Adapted Interaction, 11(1-2), 87-110.

http://dx.doi.org/10.1023/A:1011143116306

Casany, M. J., Alier, M., Mayol, E., J., P., Galanis, N., García-Peñalvo, F. J., \& Conde, M. Á. (2012). Moodbile: A Framework to Integrate m-Learning Applications with the LMS. Journal of Research and Practice in Information Technology (JRPIT), 44(2), 129-149.

Casquero, O. (2013). PLE: Una perspectiva tecnológica. In L. Castañeda \& J. Adell (Eds.), Entornos personalizados de aprendizaje: Claves para el ecosistema educativo en red (pp. 71-84). Alicante: España: Marfil.

Castaño Garrido, C., Maiz, I., \& Garay Ruiz, U. (2015). Diseño, motivación y rendimiento en un curso MOOC cooperativo. Comunicar, 44, 19-26.

http://dx.doi.org/10.3916/C44-2015-02

Centro de Nuevas Iniciativas. (2008). El conocimiento libre y los recursos educativos abiertos. Extremadura, España: Junta de Extremadura.

Chang, E., \& West, M. (2006). Digital Ecosystems A Next Generation of the Collaborative Environment. In G. Kotsis, D. Taniar, E. Pardede, \& I. K. Ibrahim (Eds.), Proceedings of iiWAS'2006 - The Eighth International Conference on Information Integration and Web-based Applications Services, 4-6 December 2006, Yogyakarta, Indonesia (pp. 3-24): Austrian Computer Society.

Chen, W., \& Chang, E. (2007). Exploring a Digital Ecosystem Conceptual Model and Its Simulation Prototype Proceedings of IEEE International Symposium on Industrial Electronics, 2007 (ISIE 2007) (pp. 2933 2938): IEEE.

Chiappe Laverde, A., Hine, N., \& Martínez Silva, J. A. (2015). Literatura y práctica: una revisión crítica acerca de los MOOC. Comunicar, 44, 09-18.

http://dx.doi.org/10.3916/C44-2015-01

Clark, D. (2013). MOOCs: taxonomy of 8 types of MOOC. Retrieved from

http://donaldclarkplanb.blogspot.com.

es/2013/04/moocs-taxonomy-of-8-types-ofmooc.html

Clark, R. C., \& Mayer, R. E. (2011). E-learning and the science of instruction: Proven guidelines for consumers and designers of multimedia learning. San Francisco, USA: Pfeiffer.

http://dx.doi.org/10.1002/9781118255971 
Collis, B. (1996). Tele-learning in a digital world. The future of distance learning. London, UK: International Thomson Computer Press.

Conde, M. Á., García-Peñalvo, F. J., Alier, M., Casany, M. J., \& Piguillem, J. (2013). Mobile devices applied to Computer Science subjects to consume institutional functionalities trough a Personal Learning Environment. International Journal of Engineering Education (IJEE), 29(3), 610619.

Conde, M. Á., García-Peñalvo, F. J., Rodríguez-Conde, M. J., Alier, M., Casany, M. J., \& Piguillem, J. (2014). An evolving Learning Management System for new educational environments using 2.0 tools. Interactive Learning Environments, 22(2), 188-204.

http://dx.doi.org/10.1080/10494820.2012.74 5433

Conde, M. Á., Muñoz, C., \& García-Peñalvo, F. J. (2008). mLearning, the First Step in the Learning Process Revolution. mLearning, the First Step in the Learning Process Revolution, 2(4), 61-63.

Conole, G. (2013a). Designing for learning in an open world. New York, NY, USA: Springer.

http://dx.doi.org/10.1007/978-1-4419-8517-0

Conole, G. (2013b). Digital identity and presence in the social milieu. Paper presented at the Pelicon conference, 2013, 10-12th April, Plymouth.

Conole, G. (2013c). Los MOOCs como tecnologías disruptivas: estrategias para mejorar la experiencia de aprendizaje y la calidad de los MOOCs. Campus Virtuales. Revista Cientifica Iberoamericana de Tecnología Educativa, 2(2), 16-28.

Conole, G. (2014). Reviewing the trajectories of e-learning. Retrieved from

http://e4innovation.com/?p=791

Conole, G., \& Alevizou, P. (2010). A literature review of the use of Web 2.0 tools in Higher Education. Retrieved January 8th, 2015, from

https://www.heacademy.ac.uk/sites/

default/files/Conole_Alevizou_2010.pdf

Corbett, A. T., Koedinger, K. R., \& Anderson, J. R. (1997). Intelligent Tutoring Systems. In M. Helander, T. K. Landauer, \& P. Prabhu (Eds.), Handbook of HumanComputer Interaction (2nd ed., pp. 849874). Amsterdam, The Netherlands: Elsevier Science.

Daniel, J. (2012). Making Sense of MOOCs: Musings in a Maze of Myth, Paradox and Possibility. Journal of Interactive Media in Education, 2012(3).

http://dx.doi.org/10.5334/2012-18

Downes, S. (2005). E-learning 2.0. eLearn Magazine(October).

Downes, S. (2012a). Connectivism and Connective Knowledge. Essays on meaning and learning networks

http://www.downes.ca.

Downes, S. (2012b). E-Learning generations. Retrieved from

http://halfanhour.blogspot.be/2012/02/elearning-generations.html

Downes, S. (2013). Week 2: The Quality of Massive Open Online Courses. Retrieved from

http://mooc.efquel.org/week-2-the-qualityof-massive-open-online-courses-by-stephendownes/

Ferguson, R. (2012). Learning analytics: Drivers, developments and challenges. International Journal of Technology Enhanced Learning, 4(5/6), 304-317.

ht t p : / / d x.doi.org / $10.1504 /$ IJTEL.2012.051816

Fidalgo Blanco, Á., García-Peñalvo, F. J., \& Sein-Echaluce Lacleta, M. L. (2013). A methodology proposal for developing adaptive cMOOC. In F. J. García-Peñalvo (Ed.), Proceedings of the First International Conference on Technological Ecosystem for Enhancing Multiculturality (TEEM'13) (pp. 
553-558). New York, NY, USA: ACM.

http://dx.doi.org/10.1145/2536536.2536621

Fidalgo Blanco, Á., Sein-Echaluce Lacleta, M. L., Borrás Gené, O., \& García-Peñalvo, F. J. (2014). Educación en abierto: Integración de un MOOC con una asignatura académica. Revista Teoría de la Educación: Educación y Cultura en la Sociedad de la Información, 15(3), 233-255.

Fidalgo Blanco, Á., Sein-Echaluce Lacleta, M. L., \& García-Peñalvo, F. J. (2013). MOOC cooperativo. Una integración entre cMOOC y xMOOC. In Á. Fidalgo Blanco \& M. L. Sein-Echaluce Lacleta (Eds.), Actas del II Congreso Internacional sobre Aprendizaje, Innovación y Competitividad (CINAIC) (pp. 481-486). Madrid, Spain: Fundación General de la Universidad Politécnica de Madrid.

Fidalgo Blanco, Á., Sein-Echaluce Lacleta, M. L., \& García-Peñalvo, F. J. (2015). Methodological Approach and Technological Framework to break the current limitations of MOOC model. Journal of Universal Computer Science, In Press.

García-Holgado, A., \& García-Peñalvo, F. J. (2013). The evolution of the technological ecosystems: An architectural proposal to enhancing learning processes. In F. J. GarcíaPeñalvo (Ed.), Proceedings of the First International Conference on Technological Ecosystems for Enhancing Multiculturality (TEEM'13) (Salamanca, Spain, November 14-15, 2013) (pp. 565-571). New York, NY, USA: ACM.

http://dx.doi.org/10.1145/2536536

http://dx.doi.org/10.1145/2536536.2536623

García-Holgado, A., \& García-Peñalvo, F. J. (2014a). Knowledge Management Ecosystem based on Drupal Platform for Promoting the Collaboration between Public Administrations. In F. J. García-Peñalvo (Ed.), Proceedings of the Second International Conference on Technological Ecosystems for Enhancing Multiculturality (TEEM'14) (Salamanca, Spain, October 1-3, 2014) (pp. 619-624). New York, NY, USA: ACM. http://dx.doi.org/10.1145/2669711.2669964 http://dx.doi.org/10.1145/2669711

García-Holgado, A., \& García-Peñalvo, F. J. (2014b). Patrón arquitectónico para la definición de ecosistemas de eLearning basados en desarrollos open source. In J. L. Sierra Rodríguez, J. M. Dodero Beardo, \& D. Burgos (Eds.), Actas del XVI Simposio Internacional de Informática Educativa (SIIE'14). Acceso masivo y universal para un aprendizaje a lo largo de la vida (Logroño, La Rioja, España, 12-14 de noviembre, 2014) (pp. 137-142).

García-Peñalvo, F. J. (2002). Software Educativo: Evolución y Tendencias. Aula. Revista de Enseñanza e Investigación Educativa, 14, 19-29.

García-Peñalvo, F. J. (2005). Estado actual de los sistemas E-Learning. Teoría de la Educación. Educación y Cultura en la Sociedad de la Información, 6(2).

García-Peñalvo, F. J. (2008). Advances in E-Learning: Experiences and Methodologies. Hershey, PA, USA: Information Science Reference (formerly Idea Group Reference).

http://dx.doi.org/10.4018/978-1-59904-756-0

García-Peñalvo, F. J. (2014). Adaptatividad y Learning Analytics: Sinergias para el futuro de la formación on-line. Retrieved Januaty 8th, 2015, from

http://gredos.usal.es/jspui/

handle/10366/125105

García-Peñalvo, F. J., Alier, M., \& Lytras, M. D. (2012). Some Reflections about Service Oriented Architectures, Cloud Computing Applications, Services and Interoperability J.UCS Special Issue. Journal of Universal Computer Science, 18(11), 1405-1409.

García-Peñalvo, F. J., Berlanga, A. J., Moreno, M. N., García-Carrasco, J., \& Carabias, J. (2004). HyCo - An authoring tool to create semantic learning objects for Web-based e-learning systems. In N. Koch, P. Fraternali, \& M. Wirsing (Eds.), Web Engineering, Proceedings (Vol. 3140, pp. 344$348)$. 
http://dx.doi.org/10.1007/978-3-540-27834$4 \_42$

García-Peñalvo, F. J., Colomo-Palacios, R., \& Lytras, M. D. (2012). Informal learning in work environments: training with the Social Web in the workplace. Behaviour 85 Information Technology, 31(8), 753-755. http://dx.doi.org/10.1080/014492 9X.2012.661548

García-Peñalvo, F. J., Conde, M. Á., Alier, M., \& Casany, M. J. (2011). Opening Learning Management Systems to Personal Learning Environments. Journal of Universal Computer Science, 17(9), 1222-1240.

http://dx.doi.org/10.3217/jucs-017-09-1222

García-Peñalvo, F. J., Cruz-Benito, J., Maderuelo, C., Pérez-Blanco, J. S., \& Martín-Suárez, A. (2014). Usalpharma: A cloud-based architecture to support Quality Assurance training processes in health area using Virtual Worlds. The Scientific World Journal, 2014.

http://dx.doi.org/10.1155/2014/659364

García-Peñalvo, F. J., Cruz-Benito, J., \& Therón, R. (2014). Visualización y Análisis de Datos en Mundos Virtuales Educativos: Comprendiendo la interacción de los usuarios en los entornos 3D. ReVisión, r(2), 46-59.

García-Peñalvo, F. J., Fidalgo Blanco, Á., \& Sein-Echaluce Lacleta, M. L. (2014). Tendencias en los MOOCs. Retrieved January 6th, 2014, from

h t t p : / / gredos.usal.es / j s p u i / handle/10366/125093

García-Peñalvo, F. J., \& García Carrasco, J. (2002). Los espacios virtuales educativos en el ámbito de Internet: Un refuerzo a la formación tradicional. Teoría de la Educación. Educación y Cultura en la Sociedad de la Información, 3.

García-Peñalvo, F. J., García de Figuerola, C., \& Merlo, J. A. (2010a). Open knowledge management in higher education. Online Information Review, 34(4), 517-519.
García-Peñalvo, F. J., García de Figuerola, C., \& Merlo, J. A. (2010b). Open knowledge: Challenges and facts. Online Information Review, 34(4), 520-539.

http://dx.doi. org/10.1108/14684521011072963

Gómez, D. A., Therón, R., \& García-Peñalvo, F. J. (2009). Semantic Spiral Timelines Used as Support for e-Learning. Journal of Universal Computer Science, 15(7), 15261545.

http://dx.doi.org/10.3217/jucs-015-07-1526

Gros, B., Lara, P., García, I., Mas, X., López, J., Maniega, D., \& Martínez, T. (2009). El modelo educativo de la UOC. Evolución y perspectivas (2nd ed.). Barcelona: Spain: Universitat Oberta de Catalunya.

Guàrdia, L., Maina, M., \& Sangrà, A. (2013). MOOC Design Principles. A Pedagogical Approach from the Learner's Perspective. eLearning Papers, 33.

Harle, J. (2008). Commonwealth libraries: Changing roles and new opportunities: Association of Commonwealth Universities.

Hirsch, B., \& Ng, J. W. P. (2011). Education beyond the cloud: Anytime-anywhere learning in a smart campus environment Proceedings of 2011 International Conference for Internet Technology and Secured Transactions (ICITST) (pp. 718 - 723): IEEE.

Hoobs, R. (2011). Digital and media literacy: Connecting culture and classroom. Thousand Oaks, CA: Corwin Press.

Illanas, A., \& Llorens, F. (2011). Los retos Web 2.0 de cara al EEES. In C. SuarezGuerrero \& F. J. García-Peñalvo (Eds.), Universidad y Desarrollo Social de la Web (pp. 13-34): Editandum.

Illanas, A., Llorens, F., Molina, R., Gallego, F., Compañ, P., Satorre, R., \& Villagrá, C. (2014). ¿Puede un videojuego ayudarnos a predecir los resultados de aprendizaje? Paper presented at the I Congreso de la Sociedad 
Española para las Ciencias del Videojuego, Barcelona, Spain.

Ingram, B. D. (2000). Scholarly rhetoric in digital media (or: Now that we have the technology, what do we do with it?). Journal of Interactive Media in Education, 3(3). http://dx.doi.org/10.5334/2000-3

International Telecommunication Union. (2014). The World in 2014: ICT Facts and Figures. from

http://www.itu.int/en/ITU-D/Statistics/ Documents/facts/ICTFactsFigures2014-e. pdf

Jordan, K. (2013). MOOC Completion Rates: The Data. Retrieved August 26, 2013, from http://www.katyjordan.com/MOOCproject. html

Kapp, K. M. (2012). The Gamification of Learning and Instruction: Game-based Methods and Strategies for Training and Education: Wiley.

Kay, J., Reimann, P., Diebold, E., \& Kummerfeld, B. (2013). MOOCs: So Many Learners, So Much Potential ... . IEEE Intelligent Systems, 28(3), 70-77.

http://dx.doi.org/10.1109/MIS.2013.66

Keim, D., Andrienko, G., Fekete, J., Görg, C., Kohlhammer, J., \& Melançon, G. (2008). Visual analytics: Definition, process, and challenges. In A. Kerren, J. Stasko, J. Fekete, \& C. North (Eds.), Information visualization (pp. 154-175). Berlin, Heidelberg: Springer. http://dx.doi.org/10.1007/978-3-540-70956$5 \_7$

Laanpere, M. (2012). Digital Learning ecosystems: rethinking virtual learning environments in the age of social media. Paper presented at the IFIP-OST'12: Open and Social Technologies for Networked Learning, Taillinn, Estonia.

Lane, L. (2012). Three Kinds of MOOCs. Retrieved from

http://lisahistory.net/wordpress/2012/08/

three-kinds-of-moocs/
Littlejohn, A. (2003). Reusing Online Resources: A Sustainable Approach to E-learning. London, UK: Routledge.

Llanas, A., Llorens, F., Molina, R., Gallego, F., Compañ, P., Satorre, R., \& Villagrá, C. (2014). ¿Puede un videojuego ayudarnos a predecir los resultados de aprendizaje? Paper presented at the I Congreso de la Sociedad Española para las Ciencias del Videojuego, Barcelona, Spain.

Llorens, F. (2009). La tecnología como motor de la innovación educativa. Estrategia y política institucional de la Universidad de Alicante. Arbor, 185(Extra), 21-32.

Llorens, F. (2011). La biblioteca universitaria como difusor de la innovación educativa. Estrategia y politica institucional de la Universidad de Alicante. Arbor, 187(Extra_3), 89-100.

Llorens, F., Molina, R., Compañ, P., \& Satorre, R. (2014). Technological Ecosystem for Open Education. In R. Neves-Silva, G. A. Tsihrintzis, V. Uskov, R. J. Howlett, \& L. C. Jain (Eds.), Smart Digital Futures 2014. Frontiers in Artificial Intelligence and Applications (Vol. 262, pp. 706-715): IOS Press.

Long, P. D., \& Siemens, G. (2011). Penetrating the Fog: Analytics in Learning and Education. EDUCAUSE Review, 46(5).

López Meneses, E., Vázquez-Cano, E., \& Román Graván, P. (2015). Análisis e implicaciones del impacto del movimiento MOOC en la comunidad científica: JCR y Scopus (2010-13). Comunicar, 44, 73-80.

http://dx.doi.org/10.3916/C44-2015-08

Margulies, A. H. (2004). A New Model for Open Sharing: Massachusetts Institute of Technology's OpenCourseWare Initiative Makes a Difference. PLoS Biology, 2(8), e200. http://dx.doi.org/10.1371/journal.

pbio.0020200

Martínez Abad, F., Rodríguez Conde, M. J., \& García-Peñalvo, F. J. (2014). Evaluación 
del impacto del término "MOOC" vs "eLearning" en la literatura científica y de divulgación. Profesorado. Revista de currículum y formación del profesorado, 18(1), 185-201.

McCormack, C., \& Jones, D. (1998). Building a Web-Based Education System. New York, NY, USA: Wiley Computer Publishing.

McGonigal, J. (2011). Reality Is Broken: Why Games Make Us Better and How They Can Change the World: Penguin Group US.

Melús-Palazón, E., Bartolomé-Moreno, C., Palacín-Arbués, J. C., Lafuente-Lafuente, A., García García, I., Guillén, Magallón-Botaya, R. (2012). Experience with using second life for medical education in a family and community medicine education unit. $B M C$ Medical Education, 12.

http://dx.doi.org/10.1186/1472-6920-12-30

Morales, E. M., García-Peñalvo, F. J., \& Barrón, Á. (2007). Improving LO quality through instructional design based on an ontological model and metadata. Journal of Universal Computer Science, 13(7), 970-979.

Morales, E. M., Gil, A. B., \& GarcíaPeñalvo, F. J. (2007). Arquitectura para la Recuperación de Objetos de Aprendizaje de Calidad en Repositorios Distribuidos. In F. Gutiérrez Vela \& P. Paderewski Rodriguez (Eds.), Actas del $5^{\circ}$ Taller en Sistemas Hipermedia Colaborativos y Adaptativos, SHCA 2007 (Vol. 1, pp. 31-38). Zaragoza, España.

Morales, E. M., Gómez-Aguilar, D., \& García-Peñalvo, F. J. (2008). HEODAR: Herramienta para la Evaluación de Objetos Didácticos de Aprendizaje Reutilizables. In J. Á. Velázquez-Iturbide, F. J. García-Peñalvo, \& A. B. Gil (Eds.), Actas del X Simposio Internacional de Informática Educativa - SIIE'08 Salamanca, España: Ediciones Universidad de Salamanca.

Morrison, D. (2003). E-learning strategies: how to get implementation and delivery right first time. New York, NY, USA: John Wiley
\& Sons, Inc.

Nkuyubwatsi, B. (2013). Evaluation of Massive Open Online Courses (MOOCs) from the learner's perspective The 12th European Conference on e-Learning ECEL-2013 (pp. 340-346): ACPI.

O'Reilly, T. (2007). What is Web 2.0: Design patterns and business models for the next generation of software. Communications \& Strategies, 1(65), 17-37.

OECD. (2007). Giving knowledge for free: The emergence of open educational resources. Paris, France: Organisation for Economic Co-operation and Development.

Open Education Consortium. (2015). Open Education Consortium. The Global Network for Open Education. Retrieved January 8th, 2015, from

http://www.oeconsortium.org/

Pata, K. (2011). Meta-design framework for open learning ecosystems. Paper presented at the Mash-UP Personal Learning Environments (MUP/PLE 2011), London, UK.

http://tihane.wordpress.com/2011/06/09/ meta-design-framework-for-open-learningecosystems-at-mupple-lecture-series/

Patel, V., Aggarwal, R., Cohen, D., Taylor, D., \& Darzi, A. (2013). Implementation of an Interactive Virtual-World Simulation for Structured Surgeon Assessment of Clinical Scenarios. Journal of the American College of Surgeons, 217(2), 270-279.

http://dx.doi.org/10.1016/j.

jamcollsurg.2013.03.023

Pew Research Center. (2014). Social Networking Fact Sheet. Retrieved December 26th, 2014, from

http://www.pewinternet.org/fact-sheets/ social-networking-fact-sheet/

Pizarro Lucas, E., Cruz-Benito, J., \& Gil Gonzalo, O. (2013). USALSIM: learning, professional practices and employability in a $3 \mathrm{D}$ virtual world. International Journal of Technology Enhanced Learning, 5(3/4), 307- 
321.

http://dx.doi.org/10.1504/

IJTEL.2013.059498

Prensky, M. (2006). "Don't bother me Mom, I'm learning!": how computer and video games are preparing your kids for twentyfirst century success and how you can help!: Paragon House.

Prensky, M. (2007). Digital Game-Based Learning: Paragon House.

Rapp, S. (2012). MOOCS \& Adaptive Learning: Spanish MOOC. Retrieved August 26, 2013, from

http://www.educause.edu/discuss/teachingand-learning/blended-and-online-learningconstituent-group/moocs-adaptive-learningspanish-mooc

Reigeluth, C. M., \& Carr-Chellman, A. A. (2009). Instructional-Design Theories and Models, Volume III: Building a Common Knowledge Base. New York, NY, USA: Taylor \& Francis.

Rosenberg, M. J. (2001). E-learning: Strategies for delivering knowledge in the digital age. New York, NY, USA: McGrawHill.

Ruipérez, G. (2003). Educación Virtual y eLearning. Madrid, Spain: Fundación Auna.

Sánchez Prieto, J. C., Olmos Migueláñez, S., \& García-Peñalvo, F. J. (2014). Understanding mobile learning: devices, pedagogical implications and research lines. Revista Teoría de la Educación: Educación y Cultura en la Sociedad de la Información, 15(1), 20-42.

Schaffert, R., \& Hilzensauer, W. (2008). On the way towards Personal Learning Environments: Seven crucial aspects. eLearning Papers, 2(9), 1-11.

SCOPEO. (2013). SCOPEO INFORME №2: MOOC: Estado de la situación actual, posibilidades, retos y futuro Scopeo Informe (Vol. 2). Salamanca, Spain: Universidad de
Salamanca.

Seoane Pardo, A. M., \& García-Peñalvo, F. J. (2014). Pedagogical Patterns and Online Teaching. In F. J. García-Peñalvo \& A. M. Seoane Pardo (Eds.), Online Tutor 2.0: Methodologies and Case Studies for Successful Learning (pp. 298-316). Hershey, PA: IGI Global.

Seoane-Pardo, A. M. (2014). Formalización de un modelo de formación online basado en el factor humano y la presencia docente mediante un lenguaje de patrón. ( $\mathrm{PhD}$ Dissertation), Universidad de Salamanca, Salamanca, Spain. Retrieved from http://gredos.usal.es/jspui/ handle/10366/123342

Seoane-Pardo, A. M., \& García-Peñalvo, F. J. (2007). Los orígenes del tutor: Fundamentos filosóficos y epistemológicos de la monitorización para su aplicación a contextos de e-learning. Teoría de la Educación: Educación y Cultura en la Sociedad de la Información, 8(2), 9-30.

Seoane-Pardo, A. M., \& García-Peñalvo, F. J. (2008). Online Tutoring and Mentoring. In G. D. Putnik \& M. M. Cunha (Eds.), Encyclopedia of Networked and Virtual Organizations (Vol. II, pp. 1120-1127). Hershey, PA, USA: Information Science Reference.

http://dx.doi.org/10.4018/978-1-59904-8857.ch147

Sharples, M., Corlett, D., \& Westmancott, O. (2002). The Design and Implementation of a Mobile Learning Resource. Personal Ubiquitous Computing, 6(3), 220-234.

http://dx.doi.org/10.1007/s007790200021

Siemens, G. (2005). Connectivism: A learning theory for the digital age. International Journal of Instructional Technology and Distance Learning, 2(1), 3-10.

Siemens, G. (2010). What are Learning Analytics? Retrieved from http://www.elearnspace.org/ blog/2010/08/25/what-are-learninganalytics/ 
Sleeman, D., \& Brown, J. S. (1982). Intelligent Tutoring Systems. London, UK: Academic Press.

Sonwalkar, N. (2013a). The First Adaptive MOOC: A Case Study on Pedagogy Framework and Scalable Cloud Architecture-Part I. MOOCs Forum, 1(P), 22-29.

http://dx.doi.org/10.1089/mooc.2013.0007

Sonwalkar, N. (2013b). MOOC: A New Frontier in Open Education. Paper presented at the United States Distance Learning Association 2013 National Conference, St. Louis, Missouri, USA.

Tan, E., \& Loughlin, E. (2014). Using 'Formally' Informal Blogs to reate Learning Communities for Students on a Teaching and Learning Programme: Peer Mentoring and Reflective Spaces. In F. J. García-Peñalvo \& A. M. Seoane-Pardo (Eds.), Online Tutor 2.0: Methodologies and Case Studies for Successful Learning (pp. 163-175). Hershey, PA: IGI Global.

http://dx.doi.org/10.4018/978-1-4666-58325.ch007

Turkle, S. (2011). Alone Together: Why We Expect More from Technology and Less from Each Other: Basic Books.

UNESCO. (2012). 2012 Paris OER Declaration. Retrieved January, 8th, 2015, from

http://www.unesco.org/new/fileadmin/ MULTIMEDIA/HQ/CI/CI/pdf/Events/

Paris OER Declaration_01.pdf

Urdan, T. A., \& Weggen, C. C. (2000). Corporate e-learning: Exploring a new frontier. San Francisco, USA: WR Hambrecht.

Warburton, S. (2009). Second Life in higher education: Assessing the potential for and the barriers to deploying virtual worlds in learning and teaching. British Journal of Educational Technology, 40(3), 414-426. http://dx.doi.org/10.1111/j.1467-

8535.2009.00952.x

Wenger, E. C., \& Snyder, W. M. (2000). Communities of Practice: The Organizational Frontier. Harvard Business Review, 78, 139145.

Werbach, K., \& Hunter, D. (2012). For the Win: How Game Thinking Can Revolutionize Your Business: Wharton Digital Press.

Wexler, S., Dublin, L., Grey, N., Jagannathan, S., Karrer, T., Martinez, M., . . . van Barneveld, A. (2007). Learning management systems. The good, the bad, the ugly,... and the truth Guild Research 360 Degree Report. Santa Rosa, California, USA: The eLearning Guild.

Wiley, D. A. (2002). Connecting learning objects to instructional design theory: A definition, a metaphor, and a taxonomy. In D. A. Wiley (Ed.), The Instructional Use of Learning Objects: Agency for Instructional Technology.

Wiley, D. A. (2008). The Learning Objects Literature. In D. Jonassen, M. J. Spector, M. Driscoll, M. D. Merrill, \& J. v. Merrienboer (Eds.), Handbook of Research for Educational Communications and Technology: A Project of the Association for Educational Communications and Technology (3rd ed., pp. 345-353). New York, NY: Routledge.

Wilson, S., Liber, O., Johnson, M., Beauvoir, P., Sharples, P., \& Milligan, C. (2007). Personal Learning Environments: Challenging the dominant design of educational systems Journal of e-Learning and Knowledge Society, $3(3), 27-38$.

Zapata-Ros, M. (2013). MOOCs, una visión crítica y una alternativa complementaria: La individualización del aprendizaje y de la ayuda pedagógica. Campus Virtuales. Revista Científica Iberoamericana de Tecnología Educativa, 2(1), 20-38. 